\title{
Visual perception and imagery: A new molecular hypothesis
}

Bókkon I.

BioSystems 96 (2009) 178-184

\begin{abstract}
Here, we put forward a redox molecular hypothesis about the natural biophysical substrate of visual perception and visual imagery. This hypothesis is based on the redox and bioluminescent processes of neuronal cells in retinotopically organized cytochrome oxidaserich visual areas. Our hypothesis is in line with the functional roles of reactive oxygen and nitrogen species in living cells that are not part of haphazard process, but rather a very strict mechanism used in signaling pathways. We point out that there is a direct relationship between neuronal activity and the biophoton emission process in the brain. Electrical and biochemical processes in the brain represent sensory information from the external world. During encoding or retrieval of information, electrical signals of neurons can be converted into synchronized biophoton signals by bioluminescent radical and non-radical processes. Therefore, information in the brain appears not only as an electrical (chemical) signal but also as a regulated biophoton (weak optical) signal inside neurons.

During visual perception, the topological distribution of photon stimuli on the retina is represented by electrical neuronal activity in retinotopically organized visual areas. These retinotopic electrical signals in visual neurons can be converted into synchronized biophoton signals by radical and non-radical processes in retinotopically organized mitochondrial-rich areas. As a result, regulated bioluminescent biophotons can create intrinsic pictures (depictive representation) in retinotopically organized cytochrome oxidase-rich visual areas during visual imagery and visual perception. The long-term visual memory is interpreted as epigenetic information regulated by free radicals and redox processes. This hypothesis does not claim to solve the secret of consciousness, but proposes that the evolution of higher levels of complexity made the intrinsic picture representation of the external visual world possible by regulated redox and bioluminescent reactions in the visual system during visual perception and visual imagery.
\end{abstract}

Keywords: Radicals as signals; Regulated biophoton emission; Retinotopically organized cytochrome oxidase (CO)-rich visual areas; Epigenetic information, Visual perception and visual imagery

\section{Introduction}

Bókkon $(2003 ; 2005 ; 2006)$ has suggested that features of the homeotherm state made the development of explicit memory possible, and that the brain can operate using pictures during informational processes (dreams, thinking) that are due to the synchronized electrical and bioluminescent biophoton processes. Recently, Bókkon (2008) has also proposed that the phosphene phenomenon is due to the intrinsic perception of induced (mechanical, electrical, magnetic, ionizing radiation, etc.) or spontaneous increased biophoton emission of cells in various parts of the visual system (from retina to cortex).

Here, we put forward a redox molecular hypothesis about visual imagery and perception. Namely, the retina converts photon signals from the external visual world into electrical signals that are carried to the striate cortex by the optic nerve. This retinotopic electrical information can be converted into regulated biophoton signals by mitochondrial redox processes that make it possible to create intrinsic pictures (depictive representation) in 
retinotopically organized cytochrome oxidase-rich visual areas during visual imagery and visual perception.

\section{Radicals as physiological second messengers in cells and the brain}

Reactive oxygen species (ROS) and reactive nitrogen species (RNS) are generated most notably by cyclooxygenases, lipoxygenases, cytochrome P450 oxidases, xanthine oxidase, NOS, the mitochondrial respiratory chain, and NADPH oxidases (Dröge 2002). ROS and RNS are traditionally viewed as dangerous byproducts of cellular metabolism. Oxidative stress occurs in biological systems when there is an overproduction of ROS/RNS or an insufficiency of enzymatic and non-enzymatic antioxidants.

Recent findings have provided evidence of the fundamental roles of ROS and RNS in intracellular signaling and intercellular communication processes (Dröge, 2002; Valko, 2007). Under physiological circumstances, ROS, and RNS can regulate gene expression, apoptosis, cell growth, cell adhesion, chemotaxis, protein-protein interactions and enzymatic functions, $\mathrm{Ca}^{2+}$ and redox homeostasis, and numerous other processes in cells (Dröge 2002, Valko, 2007; Touyz, 2005; Matsumoto et al., 2003; Devasagayam and Kamat, 2002; Gordeeva, 2006). Oxidative modifications of amino acids in proteins can serve to regulate enzyme activity (Ullrich and Kissner 2006).

A significant volume of oxygen is used to maintain neuronal membrane potentials, which subsequently produces reactive oxygen species in the brain. ROS and RNS act as signaling molecules in cerebral circulation and are necessary in molecular signal processes, synaptic plasticity, and memory formation under physiological circumstances (Ullrich and Kissner, 2006; Kishida et al., 2007; Knapp and Klann, 2002). Free radicals can control excitatory neurotransmitter glutamate uptake primarily by long-lasting oxidation of protein sulfhydryl groups in rat cortical astrocytes (Volterra et al., 1994). NADPH oxidase complex is an important source of superoxide generation that is required for normal brain function (Tejada-Simon et al., 2005). NADPH oxidase and other sources of superoxide have been implicated in hippocampal long-term potentiation (LTP) and hippocampus-dependent memory (Thiels and Klann, 2002; Thiels et al., 2000). Nitric oxide (NO) is a gaseous free radical molecule that can freely cross cell membranes and can act as a neurotransmitter or neuromodulator. Neuronal NO modulates synaptic activity by regulating neurotransmitter release. NO participates in synaptic plastic phenomena such as long-term potentiation and depression, as well as in synaptogenesis (Prast and Philippu, 2001; Holscher, 1997; Hirsch, 1993). In brief: a great number of experiments have provided strong evidence that ROS and RNS act as normal signals under physiological (pathological) circumstances in various cells and neurons.

\section{Regulated ultraweak biophoton emission is due to the bioluminescent radical and nonradical reactions in cells and neurons}

Ultraweak biophotons are continuously emitted by all living cells without any excitation (Scott et al., 1991; Quickenden and Que Hee, 1974; Yoon et al., 2005; Tilbury and Cluickenden, 1988; Kobayashi et al., 1999; Popp et al., 1984; Isojima et al., 1995; Takeda et al., 1998). Ultraweak photon emission is referred to by various names such as low-intensity chemiluminescence, dark luminescence, ultraweak electromagnetic light, ultraweak bioluminescence, ultraweak photons, biophotons etc. The ultraweak photon emission from living cells has to be differentiated from the more intensive photoemission of the luciferin/ luciferase system. 
The production of coherent and incoherent biophotons is due to the different coherent and incoherent dynamic reactions, especially bioluminescent radical and nonradical reactions of ROS and RNS and the simple cessation of excited state biomolecules. Some examples include non-enzymatic and enzymatic lipid peroxidation, mitochondrial respiration chain and peroxisomal reactions, oxidation of catecholamines, and oxidation of tyrosine and tryptophan residues in proteins, etc. (Steele, 2003; Watts et al., 1995; Kruk et al., 1989; Nakano, 2005). Mitochondrial oxidative metabolism is the main source of ultraweak photons (Thar and Kühl, 2004). This ultraweak photon emission comprises various ranges of wavelengths including infrared, visible, and ultraviolet ranges. Visible region biophoton emission is attributed to excited carbonyl groups and singlet oxygen dimers formed during the decomposition of lipid hydroperoxides (Tilbury, 1992). Moreover, chromophoric aromatic amino acids (tryptophan, tyrosine, etc.) or polyunsaturated lipids can regulate spectral visible wave-length of biophoton emission of singlet oxygen (Andersen and Lint, 1978).

We have seen that the generation of ROS and RNS is not a haphazard process, but rather a strict mechanism used in signaling pathways under physiological and pathophysiological conditions. Therefore, ultraweak biophoton emission is not a byproduct of biochemical processes but is linked to precise signaling pathways of ROS and RNS. Namely, during natural oxidative metabolism, the regulated generation of ROS and RNS can produce regulated ultraweak biophotons emission in cells.

Spontaneous biophoton emission from neural tissue depends on the neuronal membrane depolarization and $\mathrm{Ca}^{2+}$ entry into the cells (Kataoka et al., 2001). Biophoton emission can be facilitated by membrane depolarization of neurons by a high concentration of $\mathrm{K}^{+}$and can be attenuated by application of tetrodotoxin or removal of extracellular $\mathrm{Ca}^{2+}$ (Kataoka et al., 2001). According to Kobayashi et al., in vivo imaging of spontaneous biophoton emission from a rat's brain correlated with cerebral energy metabolism, EEG activity, cerebral blood flow, and oxidative stress (Kobayashi et al., 1999a, Kobayashi et al., 1999b). Biophoton emission intensity from the brain slices was dependent on temperature and oxygen concentrations. Isojima et al. (1995) reported the neural activity-dependent ultraweak biophoton emission from hippocampal slices of rat brain. Thus, we can draw a conclusion that the biophoton emission of neurons is in direct connection with biochemical processes of neurons, i.e., there is a neural activity-dependent biophoton emission in the brain. In other words, neurotransmitter-induced electrical signals (redox) of neurons can be converted into biophoton signals by bioluminescent radical and non-radical processes. Consequently, information appears not only as an electrical (chemical) signal but also as a biophoton (optical) signal in neurons (Fig. 1).

\section{Biophoton communication by photosensitive biomolecules}

Biophoton communication (as weak optical communication) of neuronal cells can be performed by natural photosensitive chromophores of neuronal cells as porphyrin ring, flavinic or pyridinic rings (Thar and Kühl, 2004). For example, the electron transport chains on the inner membrane of mitochondria contain several photosensitive chromospheres. Bioluminescent biophotons in the visible range can be absorbed by these mitochondrial chromophores. Mitochondrial cytochrome oxidase is a possible photoreceptor in mitochondria (Kato et al., 1981; Karu, 1999). Fluorescent lipid chromophores can also be photoacceptors that are formed during regulated lipid peroxidation of various membranes (Mazhul' and Shcherbin, 1999).

Photosensitive biomolecules of cells can transfer the absorbed biophoton energy to nearby biomolecules by resonance energy transfer, which can induce conformation changes 
and trigger complex signal processes in cells. Moreover, mitochondria are not only the main source of bioluminescent biophotons generated by regulated reactive oxygen species, but they also function as photoreceptors with their natural photosensitive chromophores (Kato et al., 1981; Karu, 1999). Ultraweak biophotons can be guided along the mitochondrial network (Thar and Kühl, 2004). Thus, the mitochondrial network can work as a bioluminescent biophoton communication network in cells and neurons.

We should consider that the real biophoton intensity inside mitochondria and cells can be considerably higher than one would expect from the measurements on ultraweak bioluminescence, which are generally measured macroscopically several centimetres in distance from the tissue or cell cultures. Namely, the most significant fraction of natural biophoton emission cannot be measurable or quantifiable because it is absorbed during cellular processes.

\section{Retinotopic organization, mitochondrial cytochrome oxidase-rich areas and columnar organization}

The retina converts photon signals from the external visual world into electrical signals. The lateral geniculate nucleus (LGN) receives visual information from retinal ganglion cells and sends most projections to the primary visual cortex (striate cortex, V1). Visual area V2 is the second major area in the visual cortex and the first region within the visual association area. Visual area V2 receives most of the V1 output and sends strong connections to V3, V4, and V5 (Merriam et al., 2007; Sincich and Horton, 2005). The lateral geniculate nucleus not only provides feed forward input to $\mathrm{V} 1$, but also receives robust feedback (corticogeniculate feedback) from V2 and extrastriate visual cortical areas (Briggs and Usrey, 2007). In primates, LGN, the striate cortex (V1), and many extrastriate visual cortical areas including $\mathrm{V} 2, \mathrm{~V} 3, \mathrm{~V} 4$, are organized in a retinotopic manner, respecting the topological distribution of photon stimuli on the retina (Martínez et al., 1999; Kaido et al. 2004). However, retinotopy is a fundamental organizing principle of the visual cortex. The expanded neural representation of the fovea found in the retina and LGN is maintained in the visual cortex.

One of the most persuasive examples of columnar structure is provided by the distribution of mitochondrial cytochrome oxidase in the primary visual cortex. The V1 and V2 are comprised of regions of various cytochrome oxidase (CO) activities, which can subserve different functions. In V1, layers 2 and 3 are composed of CO-dense patches (blobs) and surrounding regions (interblobs) (Xiao and Felleman, 2004). V2 is composed of alternating thin and thick CO-dense stripes and the pale interstripe regions between them. During visual perception, the high activity of cytochrome oxidase is associated with high mitochondrial activity.

Neurons tuned to different stimulus features but the same retinal positions are grouped into retinotopic arrays of hypercolumns. Hypercolumns contains three subsystems as oculardominance columns, iso-orientation domains, and blobs (Bartfeld and Grinvald, 1992). The first is the segregation of inputs from the right and the left eyes. A second system of segregated neurons is referred to as the iso-orientation domains (orientation-preference bands), each domain containing cells that respond best to a given stimulus orientation. The third subsystem contains neurons that are selective for other attributes of the visual stimulus, such as color and spatial frequency. These cells are located in the mitochondrial cytochrome oxidase-rich blobs.

Mitochondrial subunits of cytochrome oxidase activity are considered as endogenous markers of neuronal oxidative metabolism regulated by neuronal activity. The highest density 
of neurons in neocortex (number of neurons per degree of visual angle) devoted to representing the visual field is found in V1 (Rockel and Hiorns, 1980; Van Essen and Anderson, 1992). This means that the highest mitochondrial activity can be achieved in the cytochrome oxidase-rich blobs. Since V1 is retinotopic and its input is monocular, all properties must be represented for each eye. Most cells in the cytochrome oxidase-rich blobs are unoriented and selective for color, whereas most cells elsewhere are oriented and not selective for color (Lu and Roe, 2008). It was concluded that the cytochrome oxidase-rich blobs represent monocular sites of color processing in primate striate cortex. Since cytochrome oxidase is a mitochondrial respiratory chain (complex IV) enzyme, we can also use the term mitochondrial rich blobs. Therefore, mitochondrial rich blobs represent monocular sites of color processing in primate striate cortex.

Up to this point, we can summarize:

- Generation of ROS and RNS is a precise mechanism used in signaling pathways during physiological (pathological) processes in cells and neurons.

- Ultraweak photon (biophoton) emission is due to the natural reactions of reactive oxygen and nitrogen species in various cells.

- Neuronal electrical signals can be converted into biophoton (weak optical signal) signals by bioluminescent radical and non-radical processes in neurons.

- There is a neural activity-dependent ultraweak biophoton emission in the brain.

- Retinotopy is a fundamental organizing principle of the visual cortex. The expanded neural representation of the fovea found in the retina and LGN is maintained in the visual cortex.

- Most cells in the cytochrome oxidase (mitochondrial)-rich blobs are selective for color. Therefore, mitochondrial rich blobs represent monocular sites of color processing in primate striate cortex.

- The highest density of neurons in neocortex devoted to representing the visual field is found in V1. This means that the highest mitochondrial (and biophoton) activity can be achieved in the color representation cytochrome oxidase-rich blobs.

\section{Intrinsic pictures generated by bioluminescent radical and nonradical reactions in retinotopically organized mitochondrial $(\mathrm{CO})$-rich visual areas during visual perception}

Under physiological conditions, reactive oxygen and nitrogen species work as secondary messengers that is associated with biophoton emission of cells. Therefore, ultraweak photon emission of cells can also be a regulated process.

During visual perception, the topological distribution of photon stimuli on the retina is represented by electrical neuronal activity in retinotopically organized visual areas. However, activity of visual neurons is associated with their mitochondrial activity (i.e. cytochrome oxidase (CO) activity). Thus, activity of mitochondria also performs an intrinsic retinotopic representation in visual neurons.

We have seen that radical processes can produce regulated biophoton emission in cells. Therefore, electrical signals can be converted into regulated biophoton signals within cells. Besides, mitochondria are the main source of bioluminescent biophotons generated by 
regulated ROS. This means that retinotopically organized electrical patterns of neurons can also create intrinsic biophotonic patterns by the help of mitochondrial regulated ROS.

In brief: during visual perception, synchronized activation of neurons can generate intrinsic pictures by activity-dependent ultraweak photons of mitochondrial networks in retinotopically organized $\mathrm{CO}$ rich visual areas. Our hypothetical model, shown in Figure 2, is limited to a static object with a color. In reality, the computation of different kinds of visual information (motion, contrast, size, orientation, spatial frequency, texture type) is a much more complicated process.

\section{Epigenetic long-term memory}

Neuroscience regards a neuron as being a simple element whose functions are limited to the generation of electrical potentials and the transmission of signals to other neurons. According to this view, cognitive and memory functions of the nervous system are performed by neural networks consisting of simple elements. However, cognitive functions are performed by complex elements whose function is not restricted to the generation of electrical potentials and transmission of signals to other neurons. According to Arshavsky, the performance of cognitive functions is based on complex cooperative activity of "complex" neurons that are carriers of "elementary cognition" (Arshavsky, 2006).

There is increasing evidence that the regulation of chromatin structure through direct methylation of DNA or post-translational modification of histone proteins, including methylation, acetylation, and phosphorylation, can mediate long-lasting behavioral changes in the context of learning and memory (Levenson and Sweatt, 2005). DNA methylation and histone modifications work in a cooperative manner to regulate gene expression.

The latest studies suggest that epigenetic modulation of the genome is a necessary component for the formation of neuronal plasticity, associative learning and long term memory (Feng et al., 2007; Reul and Chandramohan, 2007). Chromatin structure itself can represent a "memory" and allow for temporal integration of spaced signals or metaplasticity of synapses (Levenson et al., 2004). Individual brain cells can aid in the recognition of people, landmarks, and complex pattern. Electrodes implanted deep in the brains of epileptic patients have detected single neurons dedicated to the recognition of a particular person in different situations and guises (Quiroga et al., 2005). Moreover, single cell activation is sufficient to shift the state of the entire local network (Fujisawa et al., 2006). According to the epigenetic models, cognitive and memory functions are performed not only by neural networks but also by intrinsic processes of neurons. Although the epigenetic model, which state that long term memory is stored at the level of modified DNA molecules, has obtained little recognition, this model seems to be promising. However, the synaptic plasticity/neural networks model does not contradict the epigenetic model. Neuronal networks can act as continually variable information channels among neurons, but long-term memory has a chemical/epigenetic character in individual neurons. Therefore, the epigenetic model and network model can complement each other.

\section{Long-term visual memory as redox regulated epigenetic memory}

Several authors have suggested that the long-term memory can be encoded at the level of modified DNA molecules (Feng et al., 2007; Reul and Chandramohan, 2007; Levenson et al., 2004). Roubertoux et al. provided direct evidence for the involvement of mtDNA in cognitive function (Roubertoux et al., 2003). Mitochondria have a central role in maintaining genomic stability and in controlling essential nuclear processes (Desler et al., 2007). The function of mitochondria is controlled by interactions between nuclear and mitochondrial 
genes (Roubertoux et al., 2003; Chen and Stern, 2006). Oscillations of mitochondrial energy states occur as a consequence of the interplay between mitochondrial ROS production and ROS scavenging systems in cells (Cortassa et al., 2004). Regulated production of mitochondrial free radicals can modulate gene expression processes and rewrite nuclear epigenetic information. Therefore, epigenetic long-term information can be rewritten or retrieved by mitochondrial free radicals and cellular redox processes. In our hypothetic model, long-term visual memories are not stored as images but are converted into epigenetic redox codes.

\section{Visual imagery as intrinsic pictures in the brain}

Visual mental images are constructed by activating previously stored visual information in topographically organized regions of the visual cortex (Kosslyn, 1994). In our hypothetic model, top-down processes activate and regulate the epigenetic encoded long-term visual information during visual imagery. This long-term epigenetic information can be retrieved by redox processes of cells. Then, according to retrieved epigenetic information, mitochondrial networks of neurons generate dynamic patterns of bioluminescent biophotons by regulated radical processes. Finally, synchronized dynamic patterns of bioluminescent photons can produce intrinsic pictures (depictive representation) in retinotopically organized mitochondrial (CO) rich visual neurons. Therefore, during visual imagery, we could retrieve what we thought we would have seen or done in the analogous perceptual situation. We should see that intrinsic images are not like rigid objects but we can alter images ad-lib. The important implication is that there is no a homunculus ("little men") in our head but the image representation is processed so that signals are sent to other parts of the brain, and these signals are interpreted.

\section{Visual buffer by temporary cellular redox signals and mitochondrial radicals}

A visual buffer would be used "bottom-up" to display visual perceptions and "topdown" to display internally generated images (Kosslyn, 1994). The proposed neural substrate for this buffer consists of some cortical visual areas organised retinotopically, that is, the striate and extrastriate visual areas. In our hypothesis the neural substrate of the visual buffer (a transient depository of information) is interpreted as a spatio-temporary mitochondrial redox and radical patterns that produce pulsating patterns of bioluminescent biophotons by regulated redox processes in retinotopically organized areas. These pulsating patterns of bioluminescent photons generate intrinsic pictures in retinotopically organized visual areas.

Visual perception can work as an iterative process between top-down (activated longterm visual epigenetic information) and bottom-up (from retinal map) processes as long as the perceived image and the activated long-term visual epigenetic information have a similar picture convergence. Therefore, the visual buffer makes it possible to visualize or identify an object during visual perception. However, visual imagery could, per se, use solely top-down (activated long-term visual epigenetic information) processes to display internally generated pictures in the visual buffer. Since visual imagery and perception share several functional properties and apparently share common underlying brain structures, visual imagery can interfere with visual perception (Ishai and Sagi, 1997). 


\section{Testing our hypothesis}

It is very difficult to directly test the present hypothesis, since the real biophoton intensity within cells can be considerably higher than one would expect from the measurements on ultraweak bioluminescence, which is generally measured macroscopically several centimetres in distance from the tissue or cell cultures. Namely, the most significant fraction of natural biophoton emission cannot be measurable or quantifiable because it is absorbed during cellular processes. However, if it can be proved that conscious retinotopic phosphene lights are biophotons, then intrinsic regulated biophotons could serve as a natural biophysical (redox molecular) substrate of visual perception and visual imagery.

In vitro and in vivo increases of biophoton emission should be measured in animal experiments induced by similar parameters as used in phosphene inductions by TMS (transcranial magnetic stimulation) or tDCS (transcranial direct current stimulation). It is known that hallucinogenic drugs can produce phosphenes. Therefore, in vitro and in vivo increases of biophoton emission should be measured in animal experiments produced by hallucinogenic drugs because they induce over expression of reactive and excited species, which are the main sources of biophotons. Both hallucinogenic drugs and visual mental imagery can decrease the phosphene threshold compared to control subjects. Therefore, procedures should be devised to test the TMS effect on the phosphene threshold during visual mental imagery in hallucinogenic drug users and in vivo measure of the phosphene threshold by TMS in animals' brain during administration of high dose antioxidants. Lastly, a series of other tests should be performed to compare the biophoton emission with the mitochondrial $\mathrm{CO}$, flavoprotein, and $\mathrm{NADH}$ autofluorescence activity by testing in vivo animals at the same cortical points of phosphene induction from TMS/tDCS and imagery.

\section{Summary}

We have suggested a redox molecular hypothesis of visual perception and visual imagery. This hypothesis has been based on the natural redox and bioluminescent processes of neuronal cells. We have seen that the generation of ROS and RNS is not a haphazard process, but rather a precise mechanism used in signaling pathways under physiological circumstances. Ultraweak biophotons originate from natural oxidative cell metabolism such as radical and non-radical biochemical reactions of ROS and RNS. Therefore, during natural oxidative metabolism, regulated generation of ROS and RNS is in direct connection with ultraweak photons emission of cells.

Regulated electrical (redox) signals of neurons can be converted into biophoton signals by bioluminescent radical and non-radical processes. Therefore, information in the brain appears not only as an electrical (chemical) signal but also as a regulated biophoton signal. Biophoton (as weak optical signal) communication within neuronal cells can be performed by various photosensitive biomolecules.

Mitochondrial redox processes provide the main source of ultraweak biophotons. Temporal and spatial processes of mitochondrial distribution can create temporal and spatial dynamic patterns of bioluminescent photons in neurons. Therefore, during synchronized activation of neuronal cells in retinotopically organized $\mathrm{CO}$ rich visual areas, activitydependent ultraweak photons of mitochondrial networks in space and time can create intrinsic pictures (depictive representation). This means that the retinal photon information can be represented by regulated bioluminescent photons of mitochondrial networks in retinotopically organized $\mathrm{CO}$ rich visual areas. Consequently, small clusters of visual neurons can work as "visual pixels" in accordance with the topological distribution of photon stimuli on the retina. 
Redox computation of several clusters of visual neurons can create an intrinsic picture (depictive representation) in our brain (Fig. 2).

Temporal and spatial dynamic patterns of mitochondrial free radicals can modulate gene expression processes and rewrite nuclear epigenetic information. Thus, the long-term visual memories can be interpreted as epigenetic information regulated by mitochondrial free radicals and redox processes.

During visual perception, visual redox buffer can use bottom-up and top-down processes to display visual perceptions and makes it possible to visualize or identify an object. Visual perception can work as an iterative process between the bottom-up processes (from retinal map) and the top-down (long-term epigenetic information) processes as long as the perceived picture and activated long-term epigenetic visual memories have a similar picture convergence.

During visual imagery, top-down processes activate and regulate the epigenetic encoded long-term visual information. This epigenetic information can be retrieved by redox and radical processes. Then mitochondrial networks generate dynamic patterns of bioluminescent photons by regulated redox (radical) processes. Finally, synchronized dynamic patterns of bioluminescent photons can create intrinsic pictures (as visual imageries) in retinotopically organized CO rich visual areas. Since visual imagery and perception share several functional properties and apparently share common underlying brain structures, visual imagery can interfere or facilitate visual perception.

In reality, intrinsic dynamic pictures creation by redox regulated biophotons is an extremely complex process. Moreover, visual information is linked to other sensory modalities in the brain. However, dynamic series of pictures (generated in retinotopically organized visual areas by regulated bioluminescent photons) can carry unambiguous meaning of words. The human memory can operate through intrinsic dynamic pictures and we link these pictures to each other in the learning processes.

\section{Acknowledgment}

The author Bókkon I. gratefully acknowledges support of this work by the System International Foundation (Hungary).

\section{References}

Andersen, B.R., Lint, T.F., Brendzel, A.M., 1978.Chemically shifted singlet oxygen spectrum. Biochim. Biophys. Acta 542, 527-536.

Arshavsky, Y.I., 2006. „The seven sins” of the Hebbian synapse: can the hypothesis of synaptic plasticity explain long-term memory consolidation? Prog. Neurobiol. 80, 99113.

Bartfeld, E., Grinvald, A., 1992. Relationships between orientation-preference pinwheels, cytochrome oxidase blobs, and ocular-dominance columns in primate striate cortex. PNAS 89, 1905-11909.

Bókkon, I., 2003. Creative Information. J. Biological Systems 1, 1-17.

Bókkon, I., 2005. Dreams and Neuroholography: An Interdisciplinary Interpretation of Development of Homeotherm State in Evolution. Sleep Hypnosis 7, 61-76.

Bókkon, I., 2006. Dream pictures, neuroholography and the laws of physics. J. Sleep Res. 15(Suppl. 1): 187.

Bókkon, I., 2008. Phosphene phenomenon: A new concept. BioSystems 92, 68-174. 
Briggs, F., Usrey, W.M., 2007. A fast, reciprocal pathway between the lateral geniculate nucleus and visual cortex in the macaque monkey. J. Neurosci. 27, 5431-5436.

Chen, X., Stern, D., Yan, S.D., 2006. Mitochondrial dysfunction and Alzheimer's disease. Curr. Alzheimer Res. 3, 515-520.

Cortassa, S., Aon, M.A., Winslow, R.L., O'Rourke, B., 2004. A mitochondrial oscillator dependent on reactive oxygen species. Biophys. J. 87, 2060-2073.

Desler, C., Munch-Petersen, B., Stevnsner, T., Matsui, S., Kulawiec, M., Singh, K.K., Rasmussen, L.J., 2007. Mitochondria as determinant of nucleotide pools and chromosomal stability. Mutat. Res. 625, 112-124.

Devasagayam, T.P., Kamat, J.P., 2002. Biological significance of singlet oxygen. Indian J. Exp. Biol. 40, 680-692.

Dröge, W., 2002. Free radicals in the physiological control of cell function. Physiol. Rev. 82, 47-95.

Feng, J., Fouse, S., Fan, G., 2007. Epigenetic regulation of neural gene expression and neuronal function. Pediatr. Res. 61, 58R-63R.

Fujisawa, S., Matsuki, N., Ikegaya, Y., 2006. Single neurons can induce phase transitions of cortical recurrent networks with multiple internal States. Cereb. Cortex 16, 639-654.

Gordeeva, A.V., Zvyagilskaya, R.A., Labas, Y.A., 2003. Cross-talk between reactive oxygen species and calcium in living cells. Biochemistry (Mosc) 68, 1077-1080.

Hirsch, D.B., Steiner, J.P., Dawson, T.M., Mammen, A., Hayek, E., Snyder, S.H., 1993. Neurotransmitter release regulated by nitric oxide in PC-12 cells and brain synaptosomes. Curr. Biol. 3, 749-754.

Holscher, C., 1997. Nitric oxide, the enigmatic neuronal messenger: its role in synaptic plasticity. Trends. Neurosci. 20, 298-303.

Ishai, A., Sagi, D., Visual Imagery: Effects of Short- and Long-Term Memory. 1997. J. Cogn. Neurosci. 9, 734-742.

Isojima, Y., Isoshima, T., Nagai, K., Kikuchi, K., Nakagawa, H., 1995. Ultraweak biochemiluminescence detected from rat hippocampal slices. NeuroReport 6, 658-660.

Kaido, T., Hoshida, T., Taoka, T., Sakaki, T., 2004. Retinotopy with coordinates of lateral occipital cortex in humans. J Neurosurg. 101, 114-118.

Karu, T., Primary and secondary mechanisms of action of visible to near-IR radiation on cells. 1999. J. Photochem. Photobiol. 49, 1-17.

Kataoka, Y., Cui, Y., Yamagata, A., Niigaki, M., Hirohata, T., Oishi, N., Watanabe, Y., 2001. Activity-Dependent Neural Tissue Oxidation Emits Intrinsic Ultraweak Photons. Biochem. Biophys. Res. Commun. 285, 1007-1011.

Kato, M., Shinzawa, K., Yoshikawa, S., 1981. Cytochrome oxidase is a possible photoreceptor in mitochondria. J. Photochem. Photobiol. 2, 263-269.

Kishida, K.T., Klann, E., 2007. Sources and targets of reactive oxygen species in synaptic plasticity and memory. Antioxid. Redox Signal. 9, 233-244.

Knapp, L.T., Klann, E., 2002. Role of reactive oxygen species in hippocampal long-term potentiation: contributory or inhibitory? J. Neurosci. Res. 70, 1-7.

Kobayashi, M., Takeda, M., Ito, K.I., Kato, H., Inaba, H., 1999. Two-dimensional photon counting imaging and spatiotemporal characterization of ultraweak photon emission from a rat's brain in vivo. J. Neurosci. Methods 93, 163-168.

Kobayashi, M., Takeda, M., Sato, T., Yamazaki, Y., Kaneko, K., Ito, K., Kato, H., Inaba, H., 1999. In vivo imaging of spontaneous ultraweak photon emission from a rat's brain correlated with cerebral energy metabolism and oxidative stress. Neurosci. Res. 34, $103-113$.

Kosslyn, S.M., 1994. Image and brain - The resolution of the imagery debate. MA: MIT Press, Cambridge. 
Kruk, I., Lichszteld, K., Michalska, T., Wronska, J., Bounias, M., 1989. The formation of singlet oxygen during oxidation of catechol amines as detected by infrared chemiluminescence and spectrophotometric method. Z Naturforsch. [C] 44, 895-900.

Levenson, J.M., O'Riordan, K.J., Brown, K.D., Trinh, M.A., Molfese, D.L., Sweatt, J.D., 2004. Regulation of histone acetylation during memory formation in the hippocampus. J. Biol. Chem. 279, 40545-40559.

Levenson, J.M., Sweatt, J.D., 2005. Epigenetic mechanisms in memory formation. Nat. Rev. Neurosci. 6, 108-118.

Lu, H.D., Roe, A.W., 2008. Functional organization of color domains in V1 and V2 of macaque monkey revealed by optical imaging. Cereb. Cortex 18, 516-533.

Martínez, A., Anllo-Vento, L., Sereno, M.I., Frank, L.R., Buxton, R.B., Dubowitz, D.J., Wong, E.C., Hinrichs, H., Heinze, H.J., Hillyard, S.A., 1999. Involvement of striate and extrastriate visual cortical areas in spatial attention. Nat. Neurosci. 2, 364-369.

Matsumoto, A., Comatas, K.E., Liu, L., Stamler, J.S., 2003. Screening for nitric oxidedependent protein-protein interactions. Science 301, 657-661.

Mazhul', V.M., Shcherbin, D.G., 1999. Phosphorescent analysis of lipid peroxidation products in liposomes. Biofizika. 44, 676-681.

Merriam, E.P., Genovese, C.R., Colby. C.L., 2007. Remapping in human visual cortex. J. Neurophysiol. 97, 1738-1755.

Nakano, M., 2005. Low-level chemiluminescence during lipid peroxidations and enzymatic reactions. J. Biolumin. Chemilum. 4, 231-240.

Popp, F.A., Nagl, W., Li, K.H., Scholz, W., Weingartner, O., Wolf, R., 1984. Biophoton emission. New evidence for coherence and DNA as source. Cell. Biophys. 6, 33-52.

Prast, H., Philippu, A., 2001. Nitric oxide as modulator of neuronal function. Prog. Neurobiol. $64,51-68$.

Quickenden, T.I., Que Hee, S.S., 1974. Weak luminescence from the yeast SachharomycesCervisiae. Biochem. Biophys. Res. Commun. 60, 764-770.

Quiroga, R.Q., Reddy, L., Kreiman, G., Koch, C., Fried, I., 2005. Invariant visual representation by single neurons in the human brain. Nature 435, 1102-1107.

Reul, J.M., Chandramohan, Y., 2007. Epigenetic mechanisms in stress-related memory formation. Psychoneuroendocrinology 32(Suppl. 1):S21-S25.

Rockel, A.J., Hiorns, R.W., Powell, T.P.S., 1980. The basic uniformity in structure of the neocortex. Brain 103, 221-244.

Roubertoux, P.L., Sluyter, F., Carlier, M., Marcet, B., Maarouf-Veray, F., Chérif, C., Marican, C., Arrechi, P., Godin, F., Jamon, M., Verrier, B., Cohen-Salmon, C., 2003. Mitochondrial DNA modifies cognition in interaction with the nuclear genome and age in mice. Nat. Genet. 35, 65-69.

Scott, R.Q., Roschger, P., Devaraj, B., Inaba, H., 1991. Monitoring a mammalian nuclear membrane phase transition by intrinsic ultraweak light emission. FEBS Lett. 285, 97-98.

Sincich, L.C., Horton, J.C., 2005. The circuitry of V1 and V2: integration of color, form, and motion. Annu. Rev. Neurosci. 28, 303-326.

Steele, R.H., 2003. Electromagnetic field generation by ATP-induced reverse electron transfer. Arch. Biochem. Biophys. 411, 1-18.

Takeda, M., Tanno, Y., Kobayashi, M., Usa, M., Ohuchi, N., Satomi, S., Inaba, H., 1998. A novel method of assessing carcinoma cell proliferation by biophoton emission. Cancer Lett. 127, 155-160.

Tejada-Simon, M.V., Serrano, F., Villasana, L.E., Kanterewicz, B.I., Wu, G.Y., Quinn, M.T., Klann, E., 2005. Synaptic localization of a functional NADPH oxidase in the mouse hippocampus. Mol. Cell. Neurosci. 29, 97-106. 
Thar, R., Kühl, M., 2004. Propagation of electromagnetic radiation in mitochondria? J. Theor. Biol. 230, 261-270.

Thiels, E., Klann, E., 2002. Hippocampal memory and plasticity in superoxide dismutase mutant mice. Physiol. Behav. 77, 601-605.

Thiels, E., Urban, N.N., Gonzalez-Burgos, G.R., Kanterewicz, B.I., Barrionuevo, G., Chu, C.T., Oury, T.D., Klann, E., 2000. Impairment of long-term potentiation and associative memory in mice that overexpress extracellular superoxide dismutase. J. Neurosci. 20, 7631-7639.

Tilbury, R.N., 1992. The effect of stress factors on the spontaneous photon emission from microorganisms. Experientia 48, 1030-1041.

Tilbury, R.N., Cluickenden, T.I., 1988. Spectral and time dependence studies of the ultraweak bioluminescence emitted by the bacterium Escherichia coli. Photobiochem. Photobiophys. 47, 145-150.

Touyz, R.M., 2005. Reactive oxygen species as mediators of calcium signaling by angiotensin II: implications in vascular physiology and pathophysiology. Antioxid. Redox Signal. 7, 1302-1314.

Ullrich, V., Kissner, R., 2006. Redox signaling: bioinorganic chemistry at its best. J. Inorg. Biochem. 100, 2079-2086.

Valko, M., Leibfritz, D., Moncol, J., Cronin, M.T., Mazur, M., Telser, J., 2007. Free radicals and antioxidants in normal physiological functions and human disease. Int. J. Biochem. Cell. Biol. 39, 44-84.

Van Essen, D.C., Anderson, C.H., Felleman, D.J., 1992. Information processing in the primate visual system: an integrated systems perspective. Science 255, 419-423.

Volterra, A., Trotti, D., Tromba, C., Floridi, S., Racagni, G., 1994. Glutamate uptake inhibition by oxygen free radicals in rat cortical astrocytes. J. Neurosci. 14, 2924-2932.

Watts, B.P., Barnard, M., Turrens, J.F., 1995. Peroxynitrite-Dependent Chemiluminescence of Amino Acids, Proteins, and Intact Cells. Arch. Biochem. Biophys. 317, 324-330.

Xiao, Y., Felleman, D.J., 2004. Projections from primary visual cortex to cytochrome oxidase thin stripes and interstripes of macaque visual area 2. PNAS 101, 7147-7151.

Yoon, Y.Z. Kim, J., Lee, B.C., Kim, Y.U., Lee, S.K., Soh, K.S., 2005. Changes in ultraweak photon emission and heart rate variability of epinephrine-injected rats. Gen. Physiol. Biophys. 24, 147-159. 


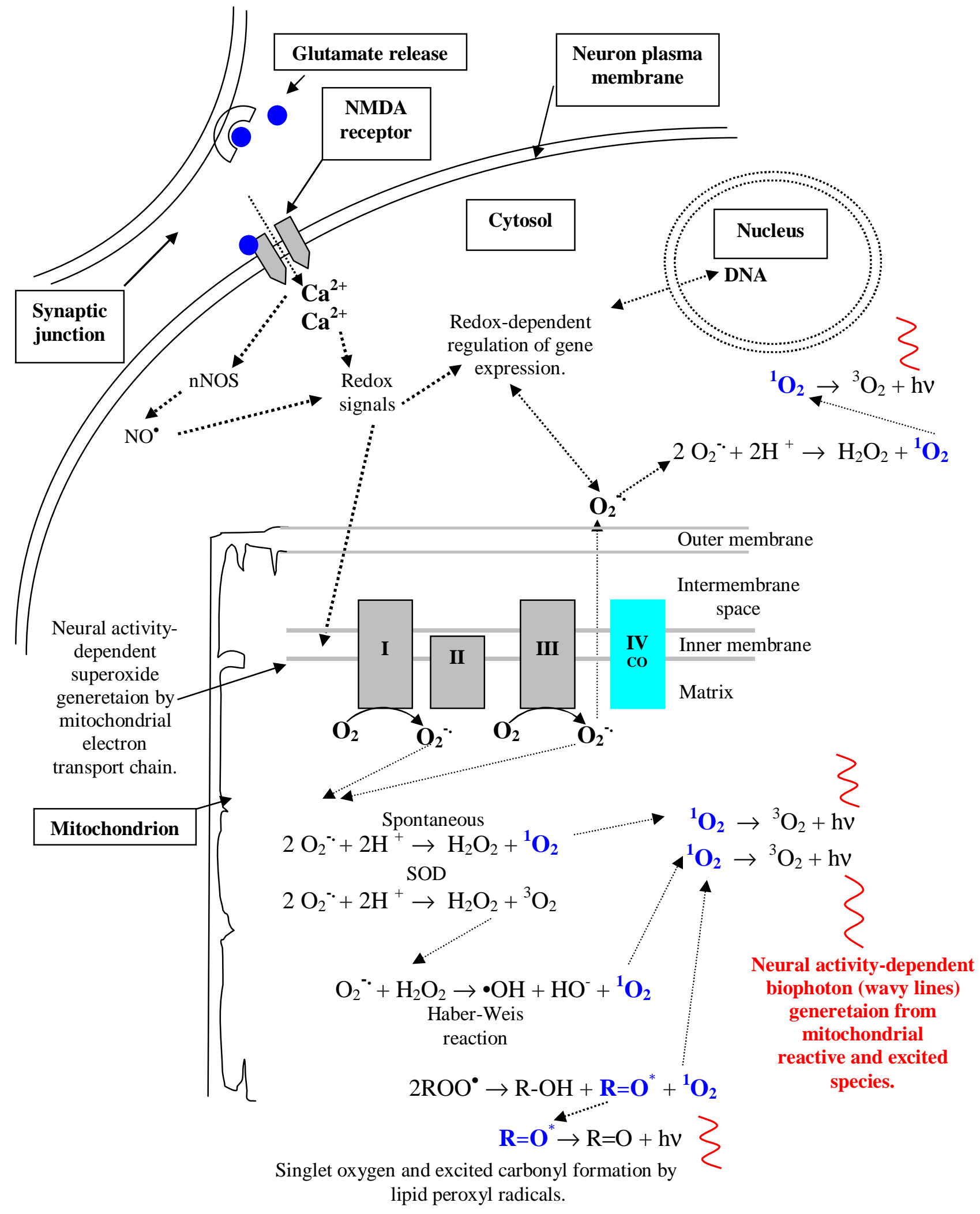

Fig. 1. Neural activity-dependent ROS and biophoton production. Activation of the NMDA receptor by glutamate induces $\mathrm{Ca}^{2+}$ influx and consequent superoxide production via activation of mitochondrial processes. Neuron activity associated with intensity of ROS and biophoton production. Mitochondrial complex IV or cytochrome oxidase (CO) is a marker of neuron activity. Mitochondrial reactions are major sources of biophoton emission. Major biophoton emission is due to the excited electrons of singlet oxygen ${ }^{1} \mathrm{O}_{2}$ and carbonyl species $\mathrm{R}=\mathrm{O}^{*}$. When an excited carbonyl or singlet oxygen is released to the ground state, it gives out its energy as a light (biophoton). 


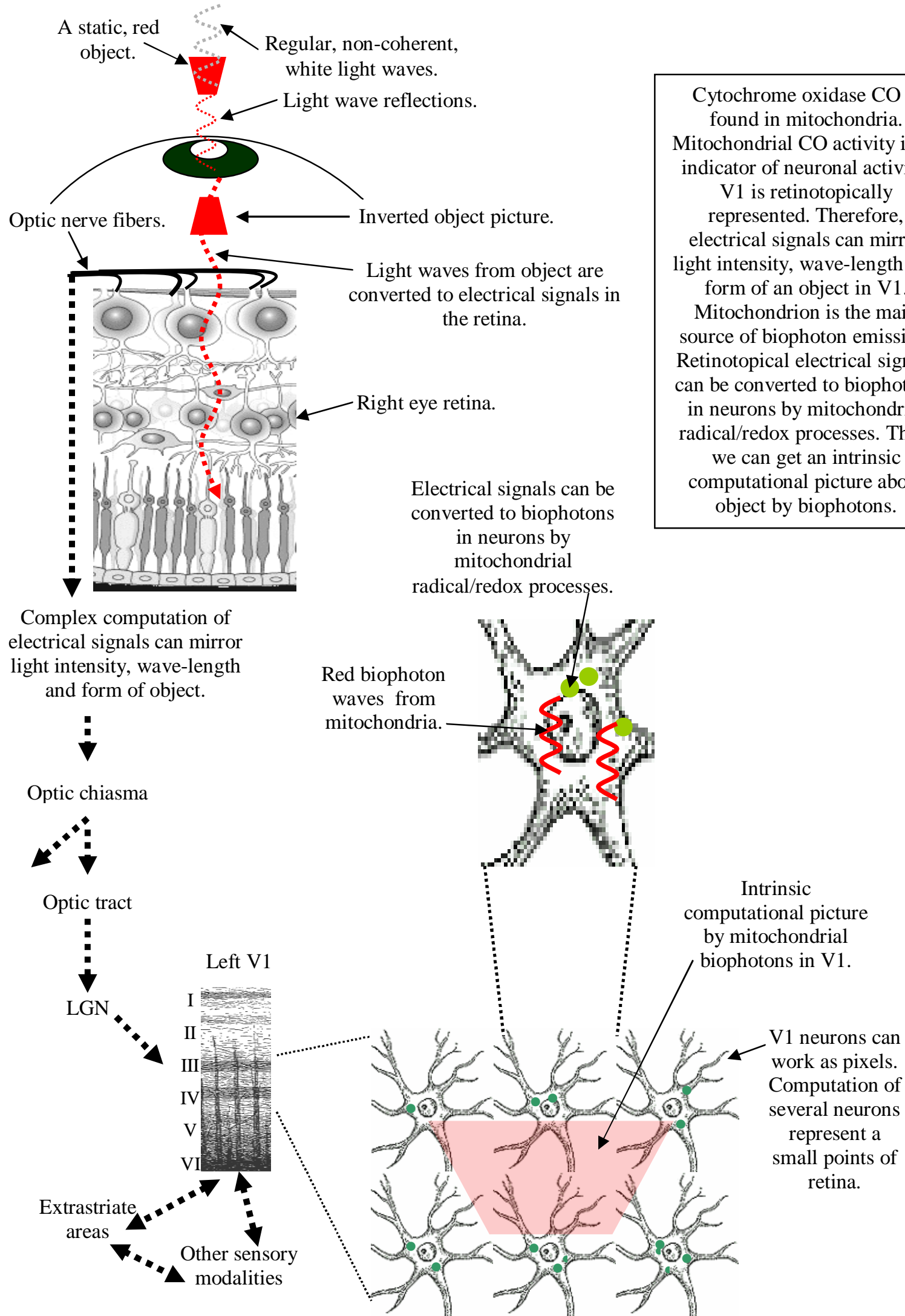

Fig. 2. Intrinsic picture representation of an object by biophotons in retinotopically organized cytochrome oxidase rich V1 areas. Light waves from object are converted to electrical signals in the retina. Retinotopical electrical signals can be converted to biophotons in V1 neurons by mitochondrial radical/redox processes. Therefore, we can get an intrinsic computational picture about object by biophotons within retinotopical V1. This hypothetic model is limited to a static object with a color. In reality, computation of different kinds of visual information (motion, contrast, size, orientation, spatial frequency, texture type) is much more complicated process. 